\title{
A PROOF OF SHELAH'S STRONG COVERING THEOREM FOR $\mathcal{P}_{\kappa} \lambda^{*}$
}

\author{
MASAHIRO SHIOYA ${ }^{\dagger}$
}

\begin{abstract}
Suppose that $\kappa$ is a regular uncountable cardinal and $\lambda$ is a cardinal $>\kappa$. We give a direct proof of Shelah's theorem that a cofinal subset of $\mathcal{P}_{\kappa} \lambda$ can be covered by some stationary set of the same size.
\end{abstract}

Key words. Stationary sets, cofinality

AMS subject classifications. 03E04, 03E05

1. Introduction. Suppose that $\kappa$ is a regular uncountable cardinal and $\lambda$ is a cardinal $\geq \kappa . \mathcal{P}_{\kappa} \lambda$ denotes the set of all subsets of $\lambda$ of size $<\kappa$. We write $\operatorname{cf}(\kappa, \lambda)$ for the cofinality of $\mathcal{P}_{\kappa} \lambda$, i.e. the smallest size of a subset of $\mathcal{P}_{\kappa} \lambda$ that is cofinal with respect to inclusion. It has been known that the formula $\lambda^{<\kappa}=2^{<\kappa} \cdot \operatorname{cf}(\kappa, \lambda)$ is useful in cardinal arithmetic. Indeed Solovay [9] established that the Singular Cardinal Hypothesis holds above a compact cardinal by showing that $\operatorname{cf}(\kappa, \lambda)=\lambda$ if $\kappa$ is $\lambda$-compact and $\lambda$ is regular.

In [6] Shelah created the Theory of Possible Cofinalities (henceforth PCF Theory). Among many applications of the theory best known should be the following:

THEOREM 1. $\operatorname{cf}\left(\omega_{1}, \omega_{\omega}\right)<\omega_{\omega_{4}}$.

Theorem 1 attracted much attention to the cofinality of $\mathcal{P}_{\kappa} \lambda$, which is quite robust through forcing extensions. In contrast it is quite easy to manipulate the size of $\mathcal{P}_{\kappa} \lambda$ $\left(=\lambda^{<\kappa}\right)$ by blowing up $2^{<\kappa}$.

In the context of $\mathcal{P}_{\kappa} \lambda$ combinatorics one is more concerned with stationary subsets than with merely cofinal subsets. Let us denote by $\operatorname{scf}(\kappa, \lambda)$ the smallest size of a stationary subset of $\mathcal{P}_{\kappa} \lambda$. Clearly $\operatorname{scf}(\kappa, \lambda) \geq \operatorname{cf}(\kappa, \lambda)$. Hence by the formula in the first paragraph we have $\operatorname{scf}(\kappa, \lambda)=\operatorname{cf}(\kappa, \lambda)=\lambda^{<\kappa}$ if $\lambda \geq 2^{<\kappa}$. In [2] Baumgartner and Taylor proved in effect that $\operatorname{scf}(\kappa, \lambda)=\operatorname{cf}(\kappa, \lambda)=\lambda$ if $\lambda<\kappa^{+\omega}$.

It is natural to ask whether $\operatorname{scf}(\kappa, \lambda)=\operatorname{cf}(\kappa, \lambda)$ holds in general. The answer by Shelah [5] is striking:

THEOREM 2. Suppose that $\kappa$ is regular uncountable and $\lambda$ is a cardinal $>\kappa$. Then $\operatorname{scf}(\kappa, \lambda)=\operatorname{cf}(\kappa, \lambda)$.

The proof of Theorem 2 makes essential use of PCF Theory. To motivate this fact, let us attempt to prove Theorem 2 by induction on $\lambda$. Suppose that for each $\gamma \in[\kappa, \lambda)$ there is a stationary subset of $\mathcal{P}_{\kappa} \gamma$ of size $\operatorname{cf}(\kappa,|\gamma|)$. Then one can show that the union of these sets is stationary in $\mathcal{P}_{\kappa} \lambda$ and has size $\operatorname{cf}(\kappa, \lambda)$ if $\lambda$ is regular. Unfortunately the induction hypothesis does not seem to help if $\lambda$ is singular. However this is exactly the situation in which PCF Theory is expected to work.

Unexpectedly the proof of Theorem 2 works equally well whenever $\lambda$ is a cardinal $>\kappa^{+}$. In [5] Shelah went on to introduce a filter on $\mathcal{P}_{\kappa} \lambda$ that contains a stationary set

\footnotetext{
*Received November 30, 2001; accepted for publication August 2, 2007.

$\dagger$ Institute of Mathematics, University of Tsukuba, Tsukuba, 305-8571 Japan (shioya@math. tsukuba.ac.jp). Partially supported by JSPS Grant-in-Aid for Scientific Research No. 16540094. The author wishes to thank Professor Shelah for answering questions at the Euresco conference "Infinite Combinatorics and Their Impact on Algebra" in Hattingen, 1999.
} 
of the smallest possible size. The definition of the filter is canonical, i.e. independent of stationary sets to be contained. It is quite complicated though.

Unfortunately the details of Shelah's proof are often missing. In $\S 4$ of this paper we give a direct and detailed proof of Theorem 2 in the case $\lambda>\kappa^{+}$. Our proof is purely combinatorial and is derived from the proof of the following theorem (again due to Shelah):

TheOREm 3. Suppose that $\kappa$ is regular uncountable and $\lambda$ is a cardinal $>\kappa$. Then a cofinal subset of $\mathcal{P}_{\kappa} \lambda$ can be covered by some stationary set of the same size.

As one might notice, Theorem 3 is no finer than Theorem 2. The point of Theorem 3 is that the way we get a covering set is canonical. This can be described as follows:

Fix a large enough regular cardinal $\chi$. Recall that $H(\chi)$ is the set of all sets hereditarily of size $<\chi$. We identify $H(\chi)$ with the structure having the membership relation and a well-ordering of $H(\chi)$ as binary relations, and $\lambda$ as a constant. Then there is a map $\varphi: \lambda^{<\omega} \rightarrow \lambda$ such that if $z \subset \lambda$ is closed under $\varphi$, then $z=N \cap \lambda$ for some $N \prec H(\chi)$. Suppose that $X$ is cofinal in $\mathcal{P}_{\kappa} \lambda$. Take an elementary substructure $M^{*}$ of $H(\chi)$ such that $X \cup\{\varphi, X\} \subset M^{*}$ and $\left|M^{*}\right|=|X|$. Then it is shown that $M^{*} \cap \mathcal{P}_{\kappa} \lambda$ is stationary in $\mathcal{P}_{\kappa} \lambda$.

The last assertion is truly remarkable. To see this, let us derive one counterintuitive consequence. Since $M^{*} \in H(\chi)$, the set $\left\{N \in \mathcal{P}_{\kappa} H(\chi): M^{*} \in N \prec H(\chi)\right\}$ is club. Hence its projection $\left\{N \cap \lambda: N \in \mathcal{P}_{\kappa} H(\chi) \wedge M^{*} \in N \prec H(\chi)\right\}$ has a subset that is club in $\mathcal{P}_{\kappa} \lambda$. Since $M^{*} \cap \mathcal{P}_{\kappa} \lambda$ is stationary in $\mathcal{P}_{\kappa} \lambda$, there is $N \prec H(\chi)$ such that $M^{*} \in N$ and $N \cap \lambda \in M^{*}$. This looks contradictory. Indeed it is easy to see that if $M$ and $N$ are both countable elementary substructures of $H(\chi)$, then one cannot have both $M \in N$ and $N \cap \lambda \in M$.

In $\S \S 2,3$ we prove Theorem 3 as outlined above. The proof of the case $\lambda>\kappa^{+}$is deeply embedded in that of Theorem 2.6 of [5]. It turns out that the latter contains an unjustifiable statement. Answering the author's question, Shelah suggested a remedy, which was to be revised by the author. We inform the reader of the important changes in the course of the proof.

In [4] Shelah initiated the study of Strong Covering Theorems. Suppose that $W$ is an inner model of ZFC and that $W \cap \mathcal{P}_{\kappa} \lambda$ is cofinal in $\mathcal{P}_{\kappa} \lambda$. Strong Covering Theorems assert under additional conditions that $W \cap \mathcal{P}_{\kappa} \lambda$ is in fact stationary. See [7] for specific examples. As indicated in [5], Theorem 3 can be regarded as the Strong Covering Theorem in the context of $\mathcal{P}_{\kappa} \lambda$ combinatorics.

2. Preliminaries. Throughout the paper $\kappa$ denotes a regular uncountable cardinal. We denote the class of all regular cardinals by Reg. Intervals of ordinals have the expected definitions. In this paper "size" is a synonym of "cardinality".

We refer the reader to [3] for the basics of $\mathcal{P}_{\kappa} \lambda$ combinatorics. Here $\lambda$ is a cardinal $>\kappa$. It is easy to see that $\bigcup X=\lambda$ if $X$ is cofinal in $\mathcal{P}_{\kappa} \lambda$. Hence $\operatorname{cf}(\kappa, \lambda) \geq \lambda$. For a map $g: \lambda^{<\omega} \rightarrow \lambda$ we denote the set $\left\{z \subset \lambda: g^{\prime \prime} z^{<\omega} \subset z\right\}$ by $C(g)$ and the closure of $z \subset \lambda$ under $g$ by $\mathrm{cl}_{g} z$. It is well-known that $S$ is stationary in $\mathcal{P}_{\kappa} \lambda$ iff $S \cap\{x \in C(g): x \cap \kappa \in \kappa\} \neq \emptyset$ for every $g: \lambda^{<\omega} \rightarrow \lambda$.

As indicated in $\S 1$, the case $\lambda=\kappa^{+}$of Theorem 2 requires a separate treatment and is proved in [2]. Let us reprove it as the case $\lambda=\kappa^{+}$of Theorem 3:

Proposition 1. Suppose that $\kappa$ is regular uncountable. Then a cofinal subset of $\mathcal{P}_{\kappa} \kappa^{+}$can be covered by some stationary set of the same size. 
Proof. Fix a large enough regular cardinal $\chi$. (It would suffice to take $\chi=\left(2^{2^{\kappa}}\right)^{+}$.) We identify $H(\chi)$ with the structure having the membership relation $\in$ and a wellordering $\triangleleft$ of $H(\chi)$ as binary relations.

Suppose that $X$ is cofinal in $\mathcal{P}_{\kappa} \kappa^{+}$. Take $M \prec H(\chi)$ so that $X \cup\{X\} \subset M$ and $|M|=|X|$. We claim that $M \cap \mathcal{P}_{\kappa} \kappa^{+}$is stationary in $\mathcal{P}_{\kappa} \kappa^{+}$. Let $e=\left\langle e_{\gamma}: \gamma<\right.$ $\left.\kappa^{+}\right\rangle$be the $\triangleleft$-least sequence such that each $e_{\gamma}$ is a surjection from $\kappa$ to $\gamma$. Then $\left\{e_{\gamma}\right.$ " $\left.\alpha: \alpha<\kappa\right\}$ is club in $\mathcal{P}_{\kappa} \gamma$ for every $\gamma \in\left[\kappa, \kappa^{+}\right)$. Hence it is easy to see that $\left\{e_{\gamma} " \alpha:\langle\alpha, \gamma\rangle \in \kappa \times \kappa^{+}\right\}$is stationary in $\mathcal{P}_{\kappa} \kappa^{+}$. Thus it suffices to show that

$$
\left\{e_{\gamma}{ }^{\prime \prime} \alpha:\langle\alpha, \gamma\rangle \in \kappa \times \kappa^{+}\right\} \subset M .
$$

Since $X \cup\{X\} \subset M \prec H(\chi)$, we have $|X|+1 \subset M$. Note that $|X| \geq \kappa^{+}$because $X$ is cofinal in $\mathcal{P}_{\kappa} \kappa^{+}$. Hence $\kappa^{+}+1 \subset M$. Since $\kappa^{+} \in M \prec H(\chi)$, we have $e \in M$. Thus we get the desired inclusion.

Toward the proof of the remaining case, we begin by quickly reviewing the basics of PCF Theory. The full theory is developed in [6], from which the following results in this section (possibly except Lemma 3) are taken. We refer the reader to [1] for an excellent introduction to the theory.

For the rest of this section we assume further $\lambda>\kappa^{+}$. Suppose that $A$ is a nonempty subset of $\left[\kappa^{++}, \lambda\right] \cap$ Reg of size $<\kappa$. We call such a set small. Note that a nonempty subset of a small set is small. Recall that

$$
\prod A=\{p: A \rightarrow \lambda: \forall \theta \in A(p(\theta)<\theta)\} .
$$

For $p, q \in \prod A$ define

$$
d(p, q)=\{\theta \in A: p(\theta)<q(\theta)\} .
$$

For $U$ an ultrafilter on $A$ define a linear ordering $<_{U}$ on $\prod A$ by:

$$
p<_{U} q \text { iff } d(p, q) \in U .
$$

We denote the cofinality of $\left(\prod A,<_{U}\right)$ by $\operatorname{cf}\left(\prod A,<_{U}\right)$. Define

$$
\operatorname{pcf} A=\left\{\operatorname{cf}\left(\prod A,<_{U}\right): U \text { is an ultrafilter on } A\right\} .
$$

We allow an ultrafilter to be principal. Hence $A \subset \operatorname{pcf} A$. It follows from Conclusion I 1.9 that $\operatorname{pcf} A$ has a maximum. Note that max $\operatorname{pcf} A$ is a regular cardinal $>\kappa^{+}$. We stipulate $\operatorname{pcf} \emptyset=\emptyset$. Finally define an ideal $J^{A}$ on $A$ by:

$$
B \in J^{A} \text { iff } \max \operatorname{pcf} B<\max \operatorname{pcf} A .
$$

Note that the definitions and results in this section are valid even if $A$ is finite, in which case ultrafilters on $A$ are all principal and hence pcf $A=A$.

Lemma 1 follows from Subfact II 3.4A (see also Lemmas I 1.5 and II 3.1).

Lemma 1. Suppose that $A$ is a small set and $\mu=\operatorname{maxpcf} A$. Then there is a scale on $A$, i.e. a sequence $\left\langle f_{\xi}: \xi<\mu\right\rangle$ of functions in $\prod A$ that is

- increasing, i.e. if $\zeta<\xi<\mu$, then $d\left(f_{\zeta}, f_{\xi}\right)$ is in the dual filter of $J^{A}$,

- cofinal, i.e. for every $p \in \prod A$ there is $\xi<\mu$ such that $d\left(p, f_{\xi}\right)=A$, and

- continuous, i.e. for every $\zeta<\mu$ of cofinality $\kappa^{+}$there is a club $D \subset \zeta$ such that if $C \subset D$ is club in $\zeta$, then $f_{\zeta}(\theta)=\sup \left\{f_{\xi}(\theta): \xi \in C\right\}$ for every $\theta \in A$. 
Lemma 2 is included in Theorem II 5.4 (see also Theorem II 3.6).

Lemma 2. Suppose that $A$ is a small set. Then $\max \operatorname{pcf} A \leq \operatorname{cf}(\kappa, \lambda)$.

Proof. Let $\mu=\operatorname{maxpcf} A$. Fix an ultrafilter $U$ on $A$ and a sequence $f=\left\langle f_{\xi}\right.$ : $\xi<\mu\rangle$ that is $<_{U}$-increasing and $<_{U}$-cofinal in $\prod A$.

Assume to the contrary $|X|<\mu$ for some cofinal $X \subset \mathcal{P}_{\kappa} \lambda$. Then for each $\xi<\mu$ there is $x \in X$ that covers $\operatorname{ran} f_{\xi} \in \mathcal{P}_{\kappa} \lambda$. Thus we get a contradiction if we find $\xi<\mu$ such that for every $x \in X$

$$
\langle\sup (x \cap \theta): \theta \in A\rangle<_{U} f_{\xi} .
$$

Let $x \in X$. Then $\langle\sup (x \cap \theta): \theta \in A\rangle \in \prod A$ because $|x|<\kappa<\theta \in$ Reg for every $\theta \in A$. Since $f$ is $<_{U}$-cofinal, there is $\xi_{x}<\mu$ such that $\langle\sup (x \cap \theta): \theta \in A\rangle<_{U} f_{\xi_{x}}$. Set $\xi=\sup \left\{\xi_{x}: x \in X\right\}$. Then $\xi<\mu$ by $|X|<\mu \in$ Reg. Since $f$ is $<_{U}$-increasing, we get the desired inequality.

We denote the set $\left\{\delta<\kappa^{+}: \operatorname{cf} \delta=\omega\right\}$ by $S_{\kappa^{+}}^{\omega}$. Shelah proved the following:

Lemma 3. Suppose that $\kappa$ is regular uncountable. Then there is a club guessing sequence on $S_{\kappa^{+}}^{\omega}$, i.e. a sequence $\left\langle c_{\delta}: \delta \in S_{\kappa^{+}}^{\omega}\right\rangle$ such that

- $c_{\delta}$ is an unbounded subset of $\delta$ of ordertype $\omega$ and

- if $E$ is club in $\kappa^{+}$, then $\left\{\delta \in S_{\kappa^{+}}^{\omega}: c_{\delta} \subset E\right\}$ is stationary in $\kappa^{+}$.

For a proof see e.g. [8]. In what follows we also invoke the proofs of Lemma II 3.4 and Observation IX 3.3A without further mention.

3. Strong Covering Theorem for $\mathcal{P}_{\kappa} \lambda$. This section is devoted to the proof of Theorem 3 in the case $\lambda>\kappa^{+}$.

THEOREM 4. Suppose that $\kappa$ is regular uncountable and $\lambda$ is a cardinal $>\kappa^{+}$. Then a cofinal subset of $\mathcal{P}_{\kappa} \lambda$ can be covered by some stationary set of the same size.

Proof. Fix a large enough regular cardinal $\chi$. (It would suffice to take $\chi=\left(2^{2^{\lambda}}\right)^{+}$.) We identify $H(\chi)$ with the structure having the membership relation $\in$ and a wellordering $\triangleleft$ of $H(\chi)$ as binary relations, and $\lambda$ as a constant. Then there is a map $\varphi:(\lambda+1)^{<\omega} \rightarrow \lambda+1$ such that

$$
C(\varphi) \subset\{N \cap(\lambda+1): N \prec H(\chi)\} .^{1}
$$

Suppose that $X$ is cofinal in $\mathcal{P}_{\kappa} \lambda$. Note that $\mathcal{P}_{\kappa} \lambda$ and $\kappa$ can be defined from $X$ in $H(\chi)$ as $\bigcup\{\mathcal{P}(x): x \in X\}$ and $\bigcup\{\mathcal{P}(x): x \in X\} \cap \lambda$ respectively. Take $M^{*} \prec H(\chi)$ so that $X \cup\{\varphi, X\} \subset M^{*}$ and $\left|M^{*}\right|=|X|$. It suffices to show the following:

MaIn Claim. $M^{*} \cap \mathcal{P}_{\kappa} \lambda$ is stationary in $\mathcal{P}_{\kappa} \lambda$.

Proof. Fix $g: \lambda^{<\omega} \rightarrow \lambda$. It suffices to find $N^{*} \prec H(\chi)$ such that $\left|N^{*}\right|<\kappa$, $N^{*} \cap \kappa \in \kappa, g \in N^{*}$ and $N^{*} \cap \lambda \in M^{*}$.

By simultaneous recursion we construct

- $N_{\gamma} \prec H(\chi)$ for $\gamma<\kappa^{+}$, and

- $N_{a}^{*} \prec H(\chi)$ for $a \in\left[\kappa^{+}\right]^{<\omega}$

so that the following hold:

(1) $\left|N_{\gamma}\right|=\kappa, N_{\gamma} \cap \kappa^{+} \in \kappa^{+},\{\varphi, X\} \subset N_{\gamma}, N_{\gamma} \cup\left\{N_{\gamma}\right\} \subset N_{\gamma+1},{ }^{2}$ $N_{\gamma}=\bigcup\left\{N_{\alpha}: \alpha<\gamma\right\}$ if $\gamma$ is limit,

\footnotetext{
${ }^{1}$ As stated in $\S 1$ we can make use of a map $\varphi: \lambda^{<\omega} \rightarrow \lambda$ (at the cost of simplicity).

${ }^{2}$ The fourth condition of (1) is redundant but is included for convenience.
} 
(2) $\left|N_{a}^{*}\right|<\kappa, N_{a}^{*} \cap \kappa \in \kappa, a \cup\{\varphi, g\} \subset N_{a}^{*}, N_{a}^{*} \cup\left\{N_{\gamma}\right\} \subset N_{a \cup\{\gamma\}}^{*}$ and

(3) $\left\langle N_{a}^{*}: a \in[\gamma+1]^{<\omega}\right\rangle \in N_{\gamma+1}$.

Set

$$
N=\bigcup\left\{N_{\gamma}: \gamma<\kappa^{+}\right\} .
$$

Then $N \prec H(\chi)$ and $|N|=\kappa^{+}$by (1). For each $\theta \in N \cap\left[\kappa^{++}, \lambda\right] \cap$ Reg, define

$$
h(\theta)=\sup (N \cap \theta) .
$$

Note that $h(\theta)<\theta$ by $|N|=\kappa^{+}<\theta \in$ Reg. Take $\alpha<\kappa^{+}$so that $\theta \in N_{\alpha}$. By (1) the map $\left\langle\sup \left(N_{\gamma} \cap \theta\right): \gamma \in\left[\alpha, \kappa^{+}\right)\right\rangle$is increasing, continuous and cofinal in $h(\theta)$. In particular $\operatorname{cf} h(\theta)=\kappa^{+}$. Similarly $\left\langle N_{\gamma} \cap \kappa^{+}: \gamma<\kappa^{+}\right\rangle$is increasing, continuous and cofinal in $\kappa^{+}$.

Let $e=\left\langle e_{\eta}: \eta \leq \lambda\right\rangle$ be the $\triangleleft$-least sequence such that each $e_{\eta}$ is an increasing, continuous and cofinal map from $\mathrm{cf} \eta$ to $\eta$. By the previous paragraph we have $e_{h(\theta)}: \kappa^{+} \rightarrow h(\theta)$ for every $\theta \in \operatorname{dom} h=N \cap\left[\kappa^{++}, \lambda\right] \cap$ Reg. Define

$$
\begin{aligned}
E=\left\{\gamma<\kappa^{+}: \gamma\right. \text { is limit } & \wedge N_{\gamma} \cap \kappa^{+}=\gamma \wedge \\
\forall \alpha & \left.<\gamma \forall \theta \in N_{\alpha} \cap\left[\kappa^{++}, \lambda\right] \cap \operatorname{Reg}\left(\sup \left(N_{\gamma} \cap \theta\right)=e_{h(\theta)}(\gamma)\right)\right\} .
\end{aligned}
$$

It is easy to see that $E$ is club in $\kappa^{+}$.

Let $c=\left\langle c_{\delta}: \delta \in S_{\kappa^{+}}^{\omega}\right\rangle$ be the $\triangleleft$-least club guessing sequence on $S_{\kappa^{+}}^{\omega}$ in the sense of Lemma 3. Take $\delta \in S_{\kappa^{+}}^{\omega} \cap E$ so that $c_{\delta} \subset E$. Define

$$
N^{*}=\bigcup\left\{N_{a}^{*}: a \in\left[c_{\delta}\right]^{<\omega}\right\} .
$$

We claim that $N^{*}$ is as required above. By (2) we have $N_{a}^{*} \subset N_{b}^{*}$ if $a \subset b$. Hence $\left\{N_{a}^{*}: a \in\left[c_{\delta}\right]^{<\omega}\right\}$ is a directed set of elementary substructures of $H(\chi)$. Thus $N^{*} \prec H(\chi)$. Since $\left|\left[c_{\delta}\right]^{<\omega}\right|=\omega<\kappa$, we have $\left|N^{*}\right|<\kappa$ and $N^{*} \cap \kappa \in \kappa$ by (2). Also $g \in N^{*}$ by (2). It remains to show that $N^{*} \cap \lambda \in M^{*}$.

Define

$$
M=\bigcup\left\{M_{a, n}: a \in\left[c_{\delta}\right]^{<\omega} \wedge n<\omega\right\},
$$

where $M_{a, n} \in \mathcal{P}_{\kappa}(\lambda+1) \cap C(\varphi)$ is defined by recursion on $n<\omega$ :

- $M_{a, 0}=\operatorname{cl}_{\varphi}\left(\left(N^{*} \cap \kappa\right) \cup a\right)$ and

- $M_{a, n+1}=$ $\operatorname{cl}_{\varphi}\left(M_{a, n} \cup\left\{e_{h(\theta)}(\gamma): \gamma \in a \wedge \theta \in \operatorname{dom} h \cap M_{a \cap \gamma, n} \cap\left[\kappa^{++}, \lambda\right] \cap \operatorname{Reg}\right\}\right) .{ }^{3}$

By definition $M_{a, n} \subset M_{a, n+1}$. By induction on $n<\omega$ we have $M_{a, n} \subset M_{b, n}$ if $a \subset b$. Hence $\left\{M_{a, n}: a \in\left[c_{\delta}\right]^{<\omega} \wedge n<\omega\right\}$ is a directed subset of $\mathcal{P}_{\kappa}(\lambda+1) \cap C(\varphi)$. Thus $M \in \mathcal{P}_{\kappa}(\lambda+1) \cap C(\varphi)$.

Claim 1. $M=N^{*} \cap(\lambda+1)$.

Proof. First we prove $M \subset N^{*}$. It suffices to show by induction on $n<\omega$ that for every $a \in\left[c_{\delta}\right]^{<\omega}$

$$
M_{a, n} \subset N^{*} \cap N_{\max a+1} \cdot{ }^{4}
$$

\footnotetext{
${ }^{3}$ The corresponding definition of $M_{y, \delta, n+1}$ in [5] is problematic.

${ }^{4}$ The corresponding induction formula (A) (a) in [5] would not work.
} 
For $n=0$, first note that $\varphi \in N^{*} \prec H(\chi)$ by (2), and that $\varphi \in N_{\max a+1} \prec H(\chi)$ by (1). Hence $N^{*}$ and $N_{\max a+1}$ are closed under $\varphi$. By the definition of $M_{a, 0}$ it suffices to show that

$$
N^{*} \cap \kappa \subset N^{*} \cap N_{\max a+1} \text { and } a \subset N^{*} \cap N_{\max a+1} .
$$

For the former, note that for every $\gamma<\kappa^{+}$

$$
\kappa \subset N_{\gamma}
$$

by $\kappa \in N_{\gamma} \cap \kappa^{+} \in \kappa^{+}$. For the latter, note that $a \subset N_{a}^{*} \subset N^{*}$ by (2) and $a \in\left[c_{\delta}\right]^{<\omega}$, and that $\max a+1 \in N_{\max a+1} \cap \kappa^{+} \in \kappa^{+}$by (3). Hence $a \subset \max a+1 \subset N_{\max a+1}$, as desired. For the induction step, first recall that $N^{*}$ and $N_{\max a+1}$ are closed under $\varphi$. By the definition of $M_{a, n+1}$ it suffices to show that if $\gamma \in a$ and $\theta \in \operatorname{dom} h \cap M_{a \cap \gamma, n} \cap$ $\left[\kappa^{++}, \lambda\right] \cap \operatorname{Reg}$, then

$$
e_{h(\theta)}(\gamma)=\sup \left(N_{\gamma} \cap \theta\right) \in N^{*} \cap N_{\max a+1} .^{5}
$$

For the equality, first note that $M_{a \cap \gamma, n} \subset N_{\max (a \cap \gamma)+1}$ by the induction hypothesis, and that $N_{\max (a \cap \gamma)+1} \subset N_{\gamma}$ by $\max (a \cap \gamma)<\gamma$. Hence $\theta \in N_{\gamma}$. Note that $\gamma$ is limit by $\gamma \in a \subset c_{\delta} \subset E$. Hence $\theta \in N_{\alpha} \cap\left[\kappa^{++}, \lambda\right] \cap$ Reg for some $\alpha<\gamma$ by (1). Thus we get the equality by $\gamma \in E$. For the membership, it suffices to show that

$$
N_{\gamma} \in N^{*} \cap N_{\max a+1} \text { and } \theta \in N^{*} \cap N_{\max a+1} .
$$

For the former, note that $N_{\gamma} \in N_{\{\gamma\}}^{*} \subset N^{*}$ by (2) and $\gamma \in c_{\delta}$, and that $N_{\gamma} \in$ $N_{\gamma+1} \subset N_{\max a+1}$ by (1) and $\gamma \leq \max a$. For the latter, recall that $\theta \in M_{a \cap \gamma, n}$ and note that $M_{a \cap \gamma, n} \subset N^{*} \cap N_{\max (a \cap \gamma)+1}$ by the induction hypothesis, and that $N_{\max (a \cap \gamma)+1} \subset N_{\max a+1}$ by $\max (a \cap \gamma)<\max a$.

Next we claim that for every $\theta \in M \cap \operatorname{Reg}$

$$
\sup (M \cap \theta)=\sup \left(N^{*} \cap \theta\right) .
$$

For $\theta \leq \kappa$, it suffices to show that

$$
M \cap \kappa \subset N^{*} \cap \kappa \subset M \cap \kappa .
$$

These follow from $M \subset N^{*}$ and $N^{*} \cap \kappa \subset M_{\emptyset, 0} \subset M$ respectively. Next suppose $\theta=\kappa^{+}$. Since $\sup c_{\delta}=\delta$, it suffices to show that

$$
c_{\delta} \subset M \cap \kappa^{+} \subset N^{*} \cap \kappa^{+} \subset N_{\delta} \cap \kappa^{+}=\delta .
$$

For the first inclusion, note that $\gamma \in M_{\{\gamma\}, 0} \subset M$ for every $\gamma \in c_{\delta}$. The next two follow from $M \subset N^{*}$ and

$$
N^{*} \subset N_{\delta}
$$

respectively. For the latter, it suffices to show that $N_{a}^{*} \subset N_{\delta}$ for every $a \in\left[c_{\delta}\right]^{<\omega}$. Recall from the previous paragraph that $a \subset N_{\max a+1}$. Since $a$ is finite, we have $a \in N_{\max a+1}$. Hence $N_{a}^{*} \in N_{\max a+1} \subset N_{\delta}$ by (3) and $\max a<\delta$. Recall that $\kappa \subset N_{\delta}$.

\footnotetext{
${ }^{5}$ Here we make an appeal to the definition of $M_{a, n+1}$ and the new induction hypothesis.
} 
Since $N_{\delta} \vDash\left|N_{a}^{*}\right|<\kappa$, we have $N_{a}^{*} \subset N_{\delta}$, as desired. The equality holds by $\delta \in E$. Finally suppose $\theta>\kappa^{+}$. It suffices to show that

$$
\sup (M \cap \theta) \leq \sup \left(N^{*} \cap \theta\right) \leq \sup \left(N_{\delta} \cap \theta\right) \leq \sup (M \cap \theta) .
$$

The first two inequalities follow from $M \subset N^{*}$ and $N^{*} \subset N_{\delta}$ respectively. For the last inequality, recall that $\theta \in M \cap \operatorname{Reg}$ and $M \subset \lambda+1$. Hence $\theta \in M \cap\left[\kappa^{++}, \lambda\right] \cap \operatorname{Reg}$. Take $a \in\left[c_{\delta}\right]^{<\omega}$ and $n<\omega$ so that $\theta \in M_{a, n}$. Since $\sup c_{\delta}=\delta$, we have $\sup \left(N_{\delta} \cap\right.$ $\theta)=\sup \left\{\sup \left(N_{\gamma} \cap \theta\right): \max a<\gamma \in c_{\delta}\right\}$ by (1). Hence it suffices to show that if $\max a<\gamma \in c_{\delta}$, then

$$
\sup \left(N_{\gamma} \cap \theta\right)=e_{h(\theta)}(\gamma) \in M .
$$

For the equality, recall from the previous paragraph that $M_{a, n} \subset N_{\max a+1}$ and note that $N_{\max a+1} \subset N_{\gamma}$ by $\max a<\gamma$. Hence $\theta \in N_{\gamma}$. Note that $\gamma$ is limit by $\gamma \in c_{\delta} \subset E$. Hence $\theta \in N_{\alpha} \cap\left[\kappa^{++}, \lambda\right] \cap$ Reg for some $\alpha<\gamma$. Thus we get the equality by $\gamma \in E$. For the membership, since $a \cup\{\gamma\} \in\left[c_{\delta}\right]^{<\omega}$, it suffices to show that

$$
e_{h(\theta)}(\gamma) \in M_{a \cup\{\gamma\}, n+1} \text {. }
$$

Recall that $\theta \in M_{a, n} \cap\left[\kappa^{++}, \lambda\right] \cap$ Reg and that $M_{a, n} \subset M \subset N^{*} \subset N_{\delta} \subset N$. Hence $\theta \in N \cap\left[\kappa^{++}, \lambda\right] \cap \operatorname{Reg}=\operatorname{dom} h$. Note that $(a \cup\{\gamma\}) \cap \gamma=a$ by $\max a<\gamma$. Hence $\theta \in M_{(a \cup\{\gamma\}) \cap \gamma, n} \cap\left[\kappa^{++}, \lambda\right] \cap$ Reg. Thus we get the membership by the definition of $M_{a \cup\{\gamma\}, n+1}$.

Finally we prove $N^{*} \cap(\lambda+1) \subset M$. Assume to the contrary that there is $\eta \in N^{*} \cap(\lambda+1)-M$. Since $M \in C(\varphi)$, there is $\bar{M} \prec H(\chi)$ such that $M=\bar{M} \cap(\lambda+1)$. Then $\lambda \in \bar{M}$ by $\bar{M} \prec H(\chi)$. Since $\eta \in(\lambda+1)-M=(\lambda+1)-\bar{M}$, we have $\eta<\lambda$. Hence we can define

$$
\bar{\eta}=\min (\bar{M} \cap[\eta, \lambda]) .
$$

Then $\bar{\eta} \in \bar{M} \cap(\lambda+1)=M \subset N^{*}$. Since $N^{*} \prec H(\chi)$, we have $\lambda \in N^{*}$ and hence $e \in N^{*}$. Thus $e_{\bar{\eta}} \in N^{*}$. Note that $\eta<\bar{\eta}$ by $\eta \notin \bar{M}$ and $\bar{\eta} \in \bar{M}$. Since $N^{*} \vDash e_{\bar{\eta}}: \operatorname{cf} \bar{\eta} \rightarrow \bar{\eta}$ is cofinal and $\eta<\bar{\eta}$, there is $\iota \in N^{*} \cap \operatorname{cf} \bar{\eta}$ such that $\eta \leq e_{\bar{\eta}}(\iota)$. Hence we get a contradiction to the minimality of $\bar{\eta}$ if we find $\bar{\iota}<\operatorname{cf} \bar{\eta}$ such that

$$
e_{\bar{\eta}}(\iota) \leq e_{\bar{\eta}}(\bar{\iota}) \in \bar{M} .
$$

Since $\bar{\eta} \in \bar{M} \cap(\lambda+1)$, we have $\operatorname{cf} \bar{\eta} \in \bar{M} \cap(\lambda+1) \cap$ Reg $=M \cap$ Reg. Hence $\sup (\bar{M} \cap \operatorname{cf} \bar{\eta})=\sup (M \cap \operatorname{cf} \bar{\eta})=\sup \left(N^{*} \cap \operatorname{cf} \bar{\eta}\right)$ by the previous paragraph. Then there is $\bar{\iota} \in \bar{M} \cap \operatorname{cf} \bar{\eta}$ such that $\iota \leq \bar{\iota}$. Since $e_{\bar{\eta}}$ is increasing, we have $e_{\bar{\eta}}(\iota) \leq e_{\bar{\eta}}(\bar{\iota})$. Since $\bar{M} \prec H(\chi)$, we have $e \in \bar{M}$ and hence $e_{\bar{\eta}}(\bar{\iota}) \in \bar{M}$ by $\bar{\eta}, \bar{\iota} \in \bar{M}$, as desired. (Claim 1)

Claim 2. $M \in M^{*}$.

Proof. It suffices to show that all the parameters needed to define the sequence $\left\langle M_{a, n}: a \in\left[c_{\delta}\right]^{<\omega} \wedge n<\omega\right\rangle$ are in $M^{*}$. By definition $\varphi \in M^{*}$. Since $X \cup\{X\} \subset M^{*} \prec$ $H(\chi)$, we have $|X| \subset M^{*}$. Note that $\lambda \leq|X|$ because $X$ is cofinal in $\mathcal{P}_{\kappa} \lambda$. Hence $\lambda \subset M^{*}$. Thus $N^{*} \cap \kappa$ and $\delta$ are in $M^{*}$. Since $\kappa, \lambda \in M^{*} \prec H(\chi), c$ and $e$ are in $M^{*}$. It remains to find $A \subset \operatorname{dom} h=N \cap\left[\kappa^{++}, \lambda\right] \cap \operatorname{Reg}$ such that $M \cap\left[\kappa^{++}, \lambda\right] \cap \operatorname{Reg} \subset A$ and $h \mid A \in M^{*}$. 
Recall that $\kappa^{+} \subset N$. Since $\kappa \in N \prec H(\chi)$, we have $c \in N$ and hence $c_{\delta} \in N$ by $\delta \in N$. Hence $\left\langle N_{a}^{*}: a \in\left[c_{\delta}\right]^{<\omega}\right\rangle \in N$ by (3). Thus $N^{*} \in N$. Note that $X \in N$ by (1). Since $N \vDash X$ is cofinal in $\mathcal{P}_{\kappa} \lambda$ and $N^{*} \cap \lambda \in \mathcal{P}_{\kappa} \lambda$, there is $x \in N \cap X$ such that $N^{*} \cap \lambda \subset x$. Define

$$
A=(x \cup\{\lambda\}) \cap\left[\kappa^{++}, \lambda\right] \cap \operatorname{Reg} .
$$

We claim that $A$ is as required above. Since $N \vDash x \in X \subset \mathcal{P}_{\kappa} \lambda$ and $\kappa \subset N$, we have $x \subset N$. Hence $A \subset N \cap\left[\kappa^{++}, \lambda\right] \cap$ Reg. Since $N^{*} \cap \lambda \subset x$, we have $M=N^{*} \cap(\lambda+1) \subset x \cup\{\lambda\}$ by Claim 1. Hence $M \cap\left[\kappa^{++}, \lambda\right] \cap \operatorname{Reg} \subset A$. It remains to show that $h \mid A \in M^{*}$.

Note that $A$ is small in the sense of $\S 2$ by $x \in \mathcal{P}_{\kappa} \lambda$ and $\kappa^{++} \in A$. Since $x \in X \subset$ $M^{*}$ and $x \in N$, we have $A \in M^{*} \cap N$.

By simultaneous recursion that terminates after finite steps, we construct

- $A_{i} \in M^{*} \cap N$ and

- $f_{i} \in M^{*} \cap \prod A_{i}$

so that the following hold for some $l<\omega$ :

- $A_{0}=A$,

- $A_{i+1} \subset A_{i}$ for every $i \leq l$,

- $h$ and $f_{i}$ agree on $A_{i}-A_{i+1}$ for every $i \leq l$, and

- $A_{l+1}=\emptyset$.

Suppose that the recursion terminates as above. Then $f_{i} \mid\left(A_{i}-A_{i+1}\right) \in M^{*}$ for every $i \leq l$. Hence we have $h \mid A=\bigcup\left\{f_{i} \mid\left(A_{i}-A_{i+1}\right): i \leq l\right\} \in M^{*}$, as desired.

Now we describe the recursion. Suppose that $A_{i} \subset A$ is defined and $A_{i} \in M^{*} \cap N$. If $A_{i}=\emptyset$, then the recursion terminates. Suppose next $A_{i} \neq \emptyset$. Then $A_{i}$ is small. We define $f_{i} \in M^{*} \cap \prod A_{i}$ and $A_{i+1} \in M^{*} \cap N$ so that $A_{i+1} \subset A_{i}$ and that $h$ and $f_{i}$ agree on $A_{i}-A_{i+1}$.

First set

$$
\mu_{i}=\max \operatorname{pcf} A_{i} .
$$

Let $f^{i}=\left\langle f_{\xi}^{i}: \xi<\mu_{i}\right\rangle$ be the $\triangleleft$-least scale on $A_{i}$ in the sense of Lemma 1. Then $\mu_{i}$ and $f^{i}$ are in $M^{*} \cap N$ by $A_{i} \in M^{*} \cap N$. Note that $\sup \left(N \cap \mu_{i}\right)<\mu_{i}$ by $|N|=\kappa^{+}<\mu_{i} \in$ Reg. Define

$$
f_{i}=f_{\sup \left(N \cap \mu_{i}\right)}^{i} .
$$

Then $f_{i} \in \prod A_{i}$. Since $A_{i}$ is small and $X$ is cofinal in $\mathcal{P}_{\kappa} \lambda$, we have $\mu_{i} \leq|X|$ by Lemma 2. Recall that $|X| \subset M^{*}$. Hence $\sup \left(N \cap \mu_{i}\right) \in M^{*}$ by $\sup \left(N \cap \mu_{i}\right)<\mu_{i}$. Since $f^{i} \in M^{*}$, we have $f_{i} \in M^{*}$.

Next we give a club $C^{*} \subset \kappa^{+}$such that for every $\theta \in A_{i}$

$$
f_{i}(\theta)=\sup \left\{f_{\sup \left(N_{\gamma} \cap \mu_{i}\right)}(\theta): \gamma \in C^{*}\right\} \leq h(\theta) .
$$

Since $A_{i} \in N$, there is $\alpha_{i}<\kappa^{+}$such that $A_{i} \in N_{\alpha_{i}}$. Then $\mu_{i} \in N_{\alpha_{i}}$. Hence by (1) the map $\left\langle\sup \left(N_{\gamma} \cap \mu_{i}\right): \gamma \in\left[\alpha_{i}, \kappa^{+}\right)\right\rangle$is increasing, continuous and cofinal in $\sup \left(N \cap \mu_{i}\right)$. In particular cf $\sup \left(N \cap \mu_{i}\right)=\kappa^{+}$. Since $f^{i}$ is continuous in $\prod A_{i}$, there is a club $D \subset \sup \left(N \cap \mu_{i}\right)$ such that if $C \subset D$ is club in $\sup \left(N \cap \mu_{i}\right)$, then $f_{i}(\theta)=$ $f_{\sup \left(N \cap \mu_{i}\right)}^{i}(\theta)=\sup \left\{f_{\xi}^{i}(\theta): \xi \in C\right\}$ for every $\theta \in A_{i}$. Take a club $C^{*} \subset\left[\alpha_{i}, \kappa^{+}\right)$so that $\left\{\sup \left(N_{\gamma} \cap \mu_{i}\right): \gamma \in C^{*}\right\} \subset D$. We claim that $C^{*}$ is as required above. For the equality, note that $\left\{\sup \left(N_{\gamma} \cap \mu_{i}\right): \gamma \in C^{*}\right\}$ is club in $\sup \left(N \cap \mu_{i}\right)$. For the inequality, 
recall that $h(\theta)=\sup (N \cap \theta)$. Hence it suffices to show that if $\theta \in A_{i}$ and $\gamma<\kappa^{+}$, then

$$
f_{\sup \left(N_{\gamma} \cap \mu_{i}\right)}^{i}(\theta) \in N .
$$

Recall that $A_{i} \in N$ and $\kappa \subset N$. Since $N \vDash\left|A_{i}\right|<\kappa$, we have $\theta \in A_{i} \subset N$. Recall that $f^{i}$ and $\mu_{i}$ are in $N$, and note that $N_{\gamma} \in N_{\gamma+1} \subset N$ by (1). Thus we get the membership.

Toward the definition of $A_{i+1}$, set

$$
\begin{aligned}
d_{i} & =d\left(f_{i}, h \mid A_{i}\right) \\
& =\left\{\theta \in A_{i}: f_{i}(\theta)<h(\theta)\right\} .
\end{aligned}
$$

Recall from the previous paragraph that $f_{i}(\theta) \leq h(\theta)$ for every $\theta \in A_{i}$. Hence $h$ and $f_{i}$ agree on $A_{i}-d_{i}$. If $d_{i}=\emptyset$, then we set $A_{i+1}=\emptyset$. Clearly $h$ and $f_{i}$ agree on $A_{i}-A_{i+1}$. Suppose next $d_{i} \neq \emptyset$. We give $\beta_{i} \in\left[\alpha_{i}, \kappa^{+}\right)$and $\xi_{i} \in N_{\beta_{i}+1} \cap \mu_{i}$ so that for every $\theta \in d_{i}$

$$
f_{i}(\theta)<\sup \left(N_{\beta_{i}} \cap \theta\right)<f_{\xi_{i}}^{i}(\theta) .
$$

Recall that $A_{i} \in N_{\alpha_{i}}$ and $\kappa \subset N_{\alpha_{i}}$. Since $N_{\alpha_{i}} \vDash\left|A_{i}\right|<\kappa$, we have $A_{i} \subset N_{\alpha_{i}}$. Hence by (1) the map $\left\langle\sup \left(N_{\gamma} \cap \theta\right): \gamma \in\left[\alpha_{i}, \kappa^{+}\right)\right\rangle$is increasing and cofinal in $\sup (N \cap \theta)=h(\theta)$ for every $\theta \in A_{i}$. Note that $\left|d_{i}\right|<\kappa$ by $d_{i} \subset A_{i} \subset A$. Hence by the definition of $d_{i}$ there is $\beta_{i} \in\left[\alpha_{i}, \kappa^{+}\right)$such that the first inequality holds for every $\theta \in d_{i}$. For the second inequality, note that $A_{i} \in N_{\alpha_{i}} \subset N_{\beta_{i}+1}$. Hence $f^{i} \in N_{\beta_{i}+1}$ and $\left\langle\sup \left(N_{\beta_{i}} \cap \theta\right): \theta \in A_{i}\right\rangle \in N_{\beta_{i}+1}$ by (1). Since $N_{\beta_{i}+1} \vDash f^{i}$ is cofinal in $\prod A_{i}$ and $\left\langle\sup \left(N_{\beta_{i}} \cap \theta\right): \theta \in A_{i}\right\rangle \in \prod A_{i}$, there is $\xi_{i} \in N_{\beta_{i}+1} \cap \mu_{i}$ such that the second inequality holds for every $\theta \in A_{i}$.

Since $C^{*}$ is club in $\kappa^{+}$, there is $\gamma_{i} \in C^{*}$ such that $\beta_{i}<\gamma_{i}$. Define

$$
\begin{aligned}
A_{i+1} & =d\left(f_{\sup \left(N_{\gamma_{i}} \cap \mu_{i}\right)}^{i}, f_{\xi_{i}}^{i}\right) \\
& =\left\{\theta \in A_{i}: f_{\sup \left(N_{\gamma_{i}} \cap \mu_{i}\right)}^{i}(\theta)<f_{\xi_{i}}^{i}(\theta)\right\} .
\end{aligned}
$$

We claim that $A_{i+1}$ is as required above. By definition $A_{i+1} \subset A_{i}$. Next we prove that $A_{i+1} \in M^{*} \cap N$. Since $f^{i} \in M^{*} \cap N$, it suffices to show that

$$
\left\{\xi_{i}, \sup \left(N_{\gamma_{i}} \cap \mu_{i}\right)\right\} \subset M^{*} \text { and }\left\{\xi_{i}, \sup \left(N_{\gamma_{i}} \cap \mu_{i}\right)\right\} \subset N .
$$

For the former, recall that $\xi_{i}$ and $\sup \left(N_{\gamma_{i}} \cap \mu_{i}\right)$ are less than $\mu_{i}$, and that $\mu_{i} \leq|X|$ and $|X| \subset M^{*}$. For the latter, recall that $\xi_{i} \in N_{\beta_{i}+1} \subset N$ and note that $N_{\gamma_{i}} \in N_{\gamma_{i}+1} \subset N$ by (1), and that $\mu_{i} \in N$. It remains to show that $h$ and $f_{i}$ agree on $A_{i}-A_{i+1}$. Since they agree on $A_{i}-d_{i}$, it suffices to show that $d_{i} \subset A_{i+1}$. We claim that for every $\theta \in d_{i}$

$$
f_{\sup \left(N_{\gamma_{i}} \cap \mu_{i}\right)}^{i}(\theta) \leq f_{i}(\theta)<f_{\xi_{i}}^{i}(\theta) .
$$

The first inequality follows from $\gamma_{i} \in C^{*}$ and $f_{i}(\theta)=\sup \left\{f_{\sup \left(N_{\gamma} \cap \mu_{i}\right)}^{i}(\theta): \gamma \in C^{*}\right\}$. The second inequality holds by the choice of $\xi_{i}$. This completes the description of the recursion.

It remains to prove that $A_{l+1}=\emptyset$ for some $l<\omega$. Assume to the contrary that $A_{i} \neq \emptyset$ for every $i<\omega$. Recall that $\xi_{i} \in N_{\beta_{i}+1} \cap \mu_{i}$ and $\beta_{i}<\gamma_{i}$. Hence $\xi_{i}<$ 
$\sup \left(N_{\beta_{i}+1} \cap \mu_{i}\right) \leq \sup \left(N_{\gamma_{i}} \cap \mu_{i}\right)$. Since $f^{i}$ is increasing in $\prod A_{i}, d\left(f_{\xi_{i}}^{i}, f_{\sup \left(N_{\gamma_{i}} \cap \mu_{i}\right)}^{i}\right)$ is in the dual filter of $J^{A_{i}}$. Hence $A_{i+1}=d\left(f_{\sup \left(N_{\gamma_{i}} \cap \mu_{i}\right)}, f_{\xi_{i}}^{i}\right) \in J^{A_{i}}$, i.e. $\max \operatorname{pcf} A_{i+1}<$ maxpcf $A_{i}$. This is the desired contradiction. प (Claim 2)

By Claims 1 and 2 we have $N^{*} \cap \lambda=M \cap \lambda \in M^{*}$, as desired. $\mathrm{Q}$ (Main Claim)

This completes the proof.

4. A direct proof of Theorem 2. This section presents a direct proof of Theorem 2 in the case $\lambda>\kappa^{+}$.

THEOREM 5. Suppose that $\kappa$ is regular uncountable and $\lambda$ is a cardinal $>\kappa^{+}$. Then $\operatorname{scf}(\kappa, \lambda)=\operatorname{cf}(\kappa, \lambda)$.

Proof. For each $\eta \leq \lambda$ fix an increasing, continuous and cofinal map $e_{\eta}$ : $\operatorname{cf} \eta \rightarrow \eta$. Take $\varphi: \lambda^{<\omega} \rightarrow \lambda$ so that if $z \in C(\varphi)$, then $e_{\eta}$ " $(z \cap \operatorname{cf} \eta)$ is an unbounded subset of $z \cap \eta$ for every $\eta \in z \cup\{\lambda\}$. For $z \subset \lambda$ set

$$
A(z)=\{\operatorname{cf} \eta: \eta \in z \cup\{\lambda\}\} \cap\left[\kappa^{++}, \lambda\right] .
$$

Note that if $x \in \mathcal{P}_{\kappa} \lambda$ and $A(x) \neq \emptyset$, then $A(x)$ is small in the sense of $\S 2$.

Suppose that $X$ is cofinal in $\mathcal{P}_{\kappa} \lambda$. We can assume that $A(x) \neq \emptyset$ for every $x \in X$. For each small set $A$ fix a scale $f^{A}=\left\langle f_{\xi}^{A}: \xi<\operatorname{maxpcf} A\right\rangle$ on $A$ in the sense of Lemma 1. For each $x \in X$ let $T(x)$ be the set of all finite sequences of small sets $\left\langle A_{i}: i \leq l\right\rangle$ such that

- $l<\omega$,

- $A_{0}=A(x)$ and

- for every $i<l$ there are $\zeta<\xi<\operatorname{maxpcf} A_{i}$ such that $A_{i+1}=d\left(f_{\xi}^{A_{i}}, f_{\zeta}^{A_{i}}\right)$. By Lemma 2 we have max pcf $A_{i} \leq|X|$ for every $i \leq l$. Hence $|T(x)| \leq|X|$.

For each $x \in X$ let $F(x)$ be the set of all functions of the form

$$
\bigcup\left\{f_{\xi_{i}}^{A_{i}} \mid\left(A_{i}-A_{i+1}\right): i \leq l\right\},
$$

where

- $\left\langle A_{i}: i \leq l\right\rangle \in T(x)$,

- $A_{l+1}=\emptyset$ and

- $\xi_{i}<\max \operatorname{pcf} A_{i}$ for every $i \leq l$.

Note that $F(x) \subset \prod A(x)$. By the previous paragraph $|F(x)| \leq|X|$.

Fix a club guessing sequence $\left\langle c_{\delta}: \delta \in S_{\kappa^{+}}^{\omega}\right\rangle$ on $S_{\kappa^{+}}^{\omega}$ in the sense of Lemma 3. Suppose that $\delta \in S_{\kappa^{+}}^{\omega}, \varepsilon<\kappa$ and $h \in F(x)$ for some $x \in X$. Define

$$
w(\delta, \varepsilon, h)=\bigcup\left\{w_{a, n}: a \in\left[c_{\delta}\right]^{<\omega} \wedge n<\omega\right\},
$$

where $w_{a, n} \in \mathcal{P}_{\kappa} \lambda \cap C(\varphi)$ is defined by recursion on $n<\omega$ :

- $w_{a, 0}=\operatorname{cl}_{\varphi}(\varepsilon \cup a)$ and

- $w_{a, n+1}=\operatorname{cl}_{\varphi}\left(w_{a, n} \cup\left\{e_{h(\theta)}(\gamma): \gamma \in a \wedge \theta \in A(x) \cap A\left(w_{a \cap \gamma, n}\right) \wedge \operatorname{cf} h(\theta)=\kappa^{+}\right\}\right)$.

By definition $w_{a, n} \subset w_{a, n+1}$. By induction on $n<\omega$ we have $w_{a, n} \subset w_{b, n}$ if $a \subset b$. Hence $\left\{w_{a, n}: a \in\left[c_{\delta}\right]^{<\omega} \wedge n<\omega\right\}$ is a directed subset of $\mathcal{P}_{\kappa} \lambda \cap C(\varphi)$. Thus $w(\delta, \varepsilon, h) \in$ $\mathcal{P}_{\kappa} \lambda \cap C(\varphi)$. Define

$$
S=\left\{w(\delta, \varepsilon, h): \delta \in S_{\kappa^{+}}^{\omega} \wedge \varepsilon<\kappa \wedge h \in \bigcup\{F(x): x \in X\}\right\} .
$$


By the previous paragraph $|S| \leq|X|$. It suffices to show the following:

Main Claim. $S$ is stationary in $\mathcal{P}_{\kappa} \lambda$.

Proof. Fix $g: \lambda^{<\omega} \rightarrow \lambda$. It suffices to find $z^{*} \in S \cap C(g)$ such that $z^{*} \cap \kappa \in \kappa$. We can assume that if $z \in C(g)$, then $z \in C(\varphi)$ and $z$ is closed under successors.

By simultaneous recursion we construct

- $z_{\gamma} \in C(g)$ for $\gamma<\kappa^{+}$,

- $z_{a}^{*} \in C(g)$ for $a \in\left[\kappa^{+}\right]<\omega$,

- $x_{\delta} \in X$ for $\delta \in S_{\kappa^{+}}^{\omega}$, and

- $\xi(A, \gamma)<\operatorname{maxpcf} A$ for $A$ a small set and $\gamma<\kappa^{+}$

so that the following hold:

(1) $\left|z_{\gamma}\right|=\kappa, \kappa \in z_{\gamma} \cap \kappa^{+} \in z_{\gamma+1} \cap \kappa^{+}, z_{\gamma} \cup\left\{\sup \left(z_{\gamma} \cap \theta\right): \theta \in A\left(z_{\gamma}\right)\right\} \subset z_{\gamma+1}$, $z_{\gamma}=\bigcup\left\{z_{\alpha}: \alpha<\gamma\right\}$ if $\gamma$ is limit,

(2) $\left|z_{a}^{*}\right|<\kappa, z_{a}^{*} \cap \kappa \in \kappa, a \subset z_{a}^{*}, z_{a}^{*} \cup\left\{\sup \left(z_{\gamma} \cap \theta\right): \theta \in A\left(z_{a}^{*}\right)\right\} \subset z_{a \cup\{\gamma\}}^{*}$,

(3) $z_{a}^{*} \subset z_{\max a+1}$,

(4) $\bigcup\left\{z_{a}^{*}: a \in\left[c_{\delta}\right]^{<\omega}\right\} \subset x_{\delta} \subset z_{\delta+1}$ if $\delta \in S_{\kappa^{+}}^{\omega}$,

(5) $\xi(A, \gamma)<\xi(A, \gamma+1), \xi(A, \gamma)=\sup \{\xi(A, \alpha): \alpha<\gamma\}$ if $\gamma$ is limit, and $\sup \left(z_{\gamma} \cap \theta\right)<f_{\xi(A, \gamma+1)}^{A}(\theta)$ for every $\theta \in A$.

(We can require (4) because $X$ is cofinal in $\mathcal{P}_{\kappa} \lambda$ and $\bigcup\left\{z_{a}^{*}: a \in\left[c_{\delta}\right]^{<\omega}\right\} \in \mathcal{P}_{\kappa} \lambda$, and (5) because $f^{A}$ is cofinal in $\prod A$ and $\left\langle\sup \left(z_{\gamma} \cap \theta\right): \theta \in A\right\rangle \in \prod A$.) We need one further requirement. For $\delta \in S_{\kappa^{+}}^{\omega}$ and $\gamma<\kappa^{+}$let $T_{\gamma}^{\delta}$ be the set of all finite sequences of small sets $\left\langle A_{i}: i \leq l\right\rangle$ such that

- $l<\omega$,

- $A_{0}=A\left(x_{\delta}\right)$ and

- for every $i<l$ there are $\alpha<\beta<\gamma$ such that $A_{i+1}=d\left(f_{\xi\left(A_{i}, \beta\right)}^{A_{i}}, f_{\xi\left(A_{i}, \alpha\right)}^{A_{i}}\right)$.

Note that $T_{\gamma}^{\delta}$ is a subset of $T\left(x_{\delta}\right)$ of size $\leq \kappa$. By definition $T_{\beta}^{\delta} \subset T_{\gamma}^{\delta}$ if $\beta<\gamma$. Finally we require that

(6) if $j<\omega, \delta \in S_{\kappa^{+}}^{\omega} \cap \gamma$ and $\left\langle A_{i}: i \leq j\right\rangle \in T_{\gamma}^{\delta}$, then $\operatorname{ran} f_{\xi\left(A_{j}, \gamma\right)}^{A_{j}} \subset z_{\gamma+1}$.

For each $\theta \in \bigcup\left\{A\left(z_{\gamma}\right): \gamma<\kappa^{+}\right\}$, define

$$
h(\theta)=\sup \left\{\sup \left(z_{\gamma} \cap \theta\right): \gamma<\kappa^{+}\right\} .
$$

Note that $h(\theta)<\theta$ by $\left|z_{\gamma}\right|<\kappa^{+}<\theta \in$ Reg. Take $\alpha<\kappa^{+}$so that $\theta \in A\left(z_{\alpha}\right)$. Note that $z_{\gamma}$ is closed under successors by $z_{\gamma} \in C(g)$. Hence $\left\langle\sup \left(z_{\gamma} \cap \theta\right): \gamma \in\left[\alpha, \kappa^{+}\right)\right\rangle$ is increasing, continuous and cofinal in $h(\theta)$ by (1). In particular $\operatorname{cf} h(\theta)=\kappa^{+}$and $e_{h(\theta)}: \kappa^{+} \rightarrow h(\theta)$. Similarly $\left\langle z_{\gamma} \cap \kappa^{+}: \gamma<\kappa^{+}\right\rangle$is increasing, continuous and cofinal in $\kappa^{+}$. Define

$E=\left\{\gamma<\kappa^{+}: \gamma\right.$ is limit $\left.\wedge z_{\gamma} \cap \kappa^{+}=\gamma \wedge \forall \alpha<\gamma \forall \theta \in A\left(z_{\alpha}\right)\left(\sup \left(z_{\gamma} \cap \theta\right)=e_{h(\theta)}(\gamma)\right)\right\}$.

It is easy to see that $E$ is club in $\kappa^{+}$.

Take $\delta \in S_{\kappa^{+}}^{\omega} \cap E$ so that $c_{\delta} \subset E$. Define

$$
z^{*}=\bigcup\left\{z_{a}^{*}: a \in\left[c_{\delta}\right]^{<\omega}\right\}
$$

We claim that $z^{*}$ is as required above. By (2) we have $z_{a}^{*} \subset z_{b}^{*}$ if $a \subset b$. Hence $\left\{z_{a}^{*}: a \in\left[c_{\delta}\right]^{<\omega}\right\}$ is a directed subset of $C(g)$. Thus $z^{*} \in C(g)$. Set

$$
\varepsilon=z^{*} \cap \kappa .
$$


Since $\left|\left[c_{\delta}\right]^{<\omega}\right|=\omega<\kappa$, we have $\varepsilon<\kappa$ by (2). It remains to show that $z^{*} \in S$. Set

$$
w=w\left(\delta, \varepsilon, h \mid A\left(x_{\delta}\right)\right) .
$$

Note that $z^{*} \subset x_{\delta} \subset z_{\delta+1}$ by (4). Hence $A\left(x_{\delta}\right) \subset \bigcup\left\{A\left(z_{\gamma}\right): \gamma<\kappa^{+}\right\}=\operatorname{dom} h$. Thus $\operatorname{dom}\left(h \mid A\left(x_{\delta}\right)\right)=A\left(x_{\delta}\right)$ and $\operatorname{cf} h(\theta)=\kappa^{+}$for every $\theta \in A\left(x_{\delta}\right)$.

Claim 1. $w=z^{*}$.

Proof. First we prove $w \subset z^{*}$. It suffices to show by induction on $n<\omega$ that for every $a \in\left[c_{\delta}\right]<\omega$

$$
w_{a, n} \subset z^{*} \cap z_{\max a+1} .
$$

For $n=0$, first note that $z^{*} \cap z_{\max a+1} \in C(g) \subset C(\varphi)$. By the definition of $w_{a, 0}$ it suffices to show that

$$
\varepsilon \subset z^{*} \cap z_{\max a+1} \text { and } a \subset z^{*} \cap z_{\max a+1} .
$$

For the former, recall that $\varepsilon=z^{*} \cap \kappa$ and note that $\kappa \subset z_{\max a+1}$ by (1). For the latter, note that $a \subset z_{a}^{*}$ by (2), and that $z_{a}^{*} \subset z^{*} \cap z_{\max a+1}$ by $a \in\left[c_{\delta}\right]^{<\omega}$ and (3). For the induction step, first recall that $z^{*} \cap z_{\max a+1} \in C(\varphi)$. By the definition of $w_{a, n+1}$ it suffices to show that if $\gamma \in a$ and $\theta \in A\left(x_{\delta}\right) \cap A\left(w_{a \cap \gamma, n}\right)$, then

$$
e_{h(\theta)}(\gamma)=\sup \left(z_{\gamma} \cap \theta\right) \in z^{*} \cap z_{\max a+1} .
$$

For the equality, first note that $w_{a \cap \gamma, n} \subset z_{\max (a \cap \gamma)+1}$ by the induction hypothesis and that $z_{\max (a \cap \gamma)+1} \subset z_{\gamma}$ by $\max (a \cap \gamma)<\gamma$. Hence $\theta \in A\left(z_{\gamma}\right)$ by $\theta \in A\left(w_{a \cap \gamma, n}\right)$. Note that $\gamma$ is limit by $\gamma \in a \subset c_{\delta} \subset E$. Hence $\theta \in A\left(z_{\alpha}\right)$ for some $\alpha<\gamma$ by (1). Thus we get the equality by $\gamma \in E$. For the membership, first note that $w_{a \cap \gamma, n} \subset z^{*}$ by the induction hypothesis. Hence $\theta \in A\left(z^{*}\right)$ by $\theta \in A\left(w_{a \cap \gamma, n}\right)$. Take $b \in\left[c_{\delta}\right]^{<\omega}$ so that $\theta \in A\left(z_{b}^{*}\right)$. Since $b \cup\{\gamma\} \in\left[c_{\delta}\right]^{<\omega}$ and $\gamma \leq \max a$, it suffices to show that

$$
\sup \left(z_{\gamma} \cap \theta\right) \in z_{b \cup\{\gamma\}}^{*} \text { and } \sup \left(z_{\gamma} \cap \theta\right) \in z_{\gamma+1} .
$$

The former follows from $\theta \in A\left(z_{b}^{*}\right)$ and (2), and the latter from $\theta \in A\left(z_{\gamma}\right)$ and (1).

Next we claim that for every $\theta \in\{\operatorname{cf} \eta: \eta \in w \cup\{\lambda\}\}$

$$
\sup (w \cap \theta)=\sup \left(z^{*} \cap \theta\right) .
$$

For $\theta \leq \kappa$, it suffices to show that

$$
w \cap \kappa \subset z^{*} \cap \kappa \subset w \cap \kappa .
$$

These follow from $w \subset z^{*}$ and $z^{*} \cap \kappa=\varepsilon \subset w_{\emptyset, 0} \subset w$ respectively. Next suppose $\theta=\kappa^{+}$. Since $\sup c_{\delta}=\delta$, it suffices to show that

$$
c_{\delta} \subset w \cap \kappa^{+} \subset z^{*} \cap \kappa^{+} \subset z_{\delta} \cap \kappa^{+}=\delta .
$$

For the first inclusion, note that $\gamma \in w_{\{\gamma\}, 0} \subset w$ for every $\gamma \in c_{\delta}$. The next two follow from $w \subset z^{*}$ and

$$
z^{*} \subset z_{\delta}
$$


respectively. For the latter, note that $z_{a}^{*} \subset z_{\max a+1} \subset z_{\delta}$ for every $a \in\left[c_{\delta}\right]^{<\omega}$ by (3). The equality holds by $\delta \in E$. Finally suppose $\theta>\kappa^{+}$. It suffices to show that

$$
\sup (w \cap \theta) \leq \sup \left(z^{*} \cap \theta\right) \leq \sup \left(z_{\delta} \cap \theta\right) \leq \sup (w \cap \theta) .
$$

The first two inequalities follow from $w \subset z^{*}$ and $z^{*} \subset z_{\delta}$ respectively. For the last inequality, first note that $\theta \in\{\operatorname{cf} \eta: \eta \in w \cup\{\lambda\}\} \cap\left[\kappa^{++}, \lambda\right]=A(w)$. Take $a \in\left[c_{\delta}\right]<\omega$ and $n<\omega$ so that $\theta \in A\left(w_{a, n}\right)$. Since $\sup c_{\delta}=\delta$, we have $\sup \left(z_{\delta} \cap \theta\right)=\sup \left\{\sup \left(z_{\gamma} \cap\right.\right.$ $\left.\theta): \max a<\gamma \in c_{\delta}\right\}$ by (1). Hence it suffices to show that if $\max a<\gamma \in c_{\delta}$, then

$$
\sup \left(z_{\gamma} \cap \theta\right)=e_{h(\theta)}(\gamma) \in w .
$$

For the equality, recall from the previous paragraph that $w_{a, n} \subset z_{\max a+1}$ and note that $z_{\max a+1} \subset z_{\gamma}$ by $\max a<\gamma$. Hence $\theta \in A\left(z_{\gamma}\right)$ by $\theta \in A\left(w_{a, n}\right)$. Note that $\gamma$ is limit by $\gamma \in c_{\delta} \subset E$. Hence $\theta \in A\left(z_{\alpha}\right)$ for some $\alpha<\gamma$ by (1). Thus we get the equality by $\gamma \in E$. For the membership, since $a \cup\{\gamma\} \in\left[c_{\delta}\right]^{<\omega}$, it suffices to show that

$$
e_{h(\theta)}(\gamma) \in w_{a \cup\{\gamma\}, n+1} .
$$

Recall that $\theta \in A\left(w_{a, n}\right)$ and that $w_{a, n} \subset w \subset z^{*} \subset x_{\delta}$. Hence $\theta \in A\left(x_{\delta}\right)$ and $\operatorname{cf} h(\theta)=\kappa^{+}$. Note that $a=(a \cup\{\gamma\}) \cap \gamma$ by max $a<\gamma$. Hence $\theta \in A\left(w_{(a \cup\{\gamma\}) \cap \gamma, n}\right)$. Thus we get the membership by the definition of $w_{a \cup\{\gamma\}, n+1}$.

Finally we prove $z^{*} \subset w$. Assume to the contrary that there is $\eta \in z^{*}-w$. Then $\eta<\lambda$ by $z^{*} \subset \lambda$. Hence we can define

$$
\bar{\eta}=\min ((w \cup\{\lambda\})-\eta) .
$$

Note that $\eta \in \lambda-w$ and $\bar{\eta} \in w \cup\{\lambda\}$. Hence $\eta<\bar{\eta}$ by $\eta \leq \bar{\eta}$, and $\bar{\eta} \in z^{*} \cup\{\lambda\}$ by $w \subset z^{*}$. Since $z^{*} \in C(g) \subset C(\varphi), e_{\bar{\eta}}$ " $\left(z^{*} \cap \operatorname{cf} \bar{\eta}\right)$ is unbounded in $z^{*} \cap \bar{\eta}$ by the choice of $\varphi$. Hence there is $\iota \in z^{*} \cap \operatorname{cf} \bar{\eta}$ such that $\eta \leq e_{\bar{\eta}}(\iota)$. Thus we get a contradiction to the minimality of $\bar{\eta}$ if we find $\bar{\imath}<\operatorname{cf} \bar{\eta}$ such that

$$
e_{\bar{\eta}}(\iota) \leq e_{\bar{\eta}}(\bar{\iota}) \in w .
$$

Since $\bar{\eta} \in w \cup\{\lambda\}$, we have $\sup (w \cap \operatorname{cf} \bar{\eta})=\sup \left(z^{*} \cap \operatorname{cf} \bar{\eta}\right)$ by the previous paragraph. Hence there is $\bar{\iota} \in w \cap \mathrm{cf} \bar{\eta}$ such that $\iota \leq \bar{\iota}$. Since $e_{\bar{\eta}}$ is increasing, we have $e_{\bar{\eta}}(\iota) \leq e_{\bar{\eta}}(\bar{l})$. Recall that $w \in C(\varphi)$ and that $\bar{\eta} \in w \cup\{\lambda\}$ and $\bar{\iota} \in w \cap \operatorname{cf} \bar{\eta}$. Hence $e_{\bar{\eta}}(\bar{\iota}) \in w$, as desired. $\mathrm{Q}$ (Claim 1)

Claim 2. $w \in S$.

Proof. Recall that $w=w\left(\delta, \varepsilon, h \mid A\left(x_{\delta}\right)\right)$. It suffices to show that $h \mid A\left(x_{\delta}\right) \in F\left(x_{\delta}\right)$.

By simultaneous recursion that terminates after finite steps, we construct

- $A_{j} \subset A\left(x_{\delta}\right)$ and

- $\xi_{j}<\max \operatorname{pcf} A_{j}$

so that the following hold for some $l<\omega$ :

- $A_{0}=A\left(x_{\delta}\right)$,

- for every $j \leq l$ there is $\gamma<\kappa^{+}$such that $\left\langle A_{i}: i \leq j\right\rangle \in T_{\gamma}^{\delta}$,

- $h$ and $f_{\xi_{j}}^{A_{j}}$ agree on $A_{j}-A_{j+1}$ for every $j \leq l$, and

- $A_{l+1}=\emptyset$. 
Suppose that the recursion terminates as above. Recall that $T_{\gamma}^{\delta} \subset T\left(x_{\delta}\right)$ for every $\gamma<\kappa^{+}$. Hence $\left\langle A_{i}: i \leq l\right\rangle \in T\left(x_{\delta}\right)$. Thus $h \mid A\left(x_{\delta}\right)=\bigcup\left\{f_{\xi_{i}}^{A_{i}} \mid\left(A_{i}-A_{i+1}\right): i \leq l\right\} \in$ $F\left(x_{\delta}\right)$, as desired.

Now we describe the recursion. Suppose that $\left\langle A_{i}: i \leq j\right\rangle$ is defined. If $A_{j}=\emptyset$, then the recursion terminates. Suppose next $A_{j} \neq \emptyset$. By the induction hypothesis there is $\alpha_{j} \in\left(\delta, \kappa^{+}\right)$such that $\left\langle A_{i}: i \leq j\right\rangle \in T_{\alpha_{j}}^{\delta}$. Define

$$
\xi_{j}=\sup \left\{\xi\left(A_{j}, \gamma\right): \gamma<\kappa^{+}\right\} .
$$

Then $\xi_{j}<\operatorname{maxpcf} A_{j}$ by $\kappa^{+}<\operatorname{maxpcf} A_{j} \in$ Reg. We define $A_{j+1} \subset A_{j}$ so that $h$ and $f_{\xi_{j}}^{A_{j}}$ agree on $A_{j}-A_{j+1}$ and that if $A_{j+1} \neq \emptyset$, then $\left\langle A_{i}: i \leq j+1\right\rangle \in T_{\gamma}^{\delta}$ for some $\gamma<\kappa^{+}$.

First we give a club $C^{*} \subset \kappa^{+}$such that for every $\theta \in A_{j}$

$$
f_{\xi_{j}}^{A_{j}}(\theta)=\sup \left\{f_{\xi\left(A_{j}, \gamma\right)}^{A_{j}}(\theta): \gamma \in C^{*}\right\} \leq h(\theta) .
$$

By (5) the map $\left\langle\xi\left(A_{j}, \gamma\right): \gamma<\kappa^{+}\right\rangle$is increasing, continuous and cofinal in $\sup \left\{\xi\left(A_{j}, \gamma\right): \gamma<\kappa^{+}\right\}=\xi_{j}$. In particular $\operatorname{cf} \xi_{j}=\kappa^{+}$. Since $f^{A_{j}}$ is continuous in $\prod A_{j}$, there is a club $D \subset \xi_{j}$ such that if $C \subset D$ is club in $\xi_{j}$, then $f_{\xi_{j}}^{A_{j}}(\theta)=\sup \left\{f_{\xi}^{A_{j}}(\theta): \xi \in C\right\}$ for every $\theta \in A_{j}$. Take a club $C^{*} \subset\left[\alpha_{j}, \kappa^{+}\right)$so that $\left\{\xi\left(A_{j}, \gamma\right): \gamma \in C^{*}\right\} \subset D$. We claim that $C^{*}$ is as required above. For the equality, note that $\left\{\xi\left(A_{j}, \gamma\right): \gamma \in C^{*}\right\}$ is club in $\xi_{j}$. For the inequality, recall that $h(\theta)=\sup \left\{\sup \left(z_{\gamma} \cap \theta\right): \gamma<\kappa^{+}\right\}$. Hence it suffices to show that if $\theta \in A_{j}$ and $\gamma \in C^{*}$, then

$$
f_{\xi\left(A_{j}, \gamma\right)}^{A_{j}}(\theta) \in z_{\gamma+1}
$$

Recall that $\left\langle A_{i}: i \leq j\right\rangle \in T_{\alpha_{j}}^{\delta}$ and note that $\alpha_{j} \leq \gamma$ by $C^{*} \subset\left[\alpha_{j}, \kappa^{+}\right)$. Hence $\left\langle A_{i}: i \leq j\right\rangle \in T_{\gamma}^{\delta}$. Note that $\delta<\gamma$ by $\delta<\alpha_{j}$. Thus we get the membership by (6).

Toward the definition of $A_{j+1}$, set

$$
\begin{aligned}
d_{j} & =d\left(f_{\xi_{j}}^{A_{j}}, h \mid A_{j}\right) \\
& =\left\{\theta \in A_{j}: f_{\xi_{j}}^{A_{j}}(\theta)<h(\theta)\right\} .
\end{aligned}
$$

Recall from the previous paragraph that $f_{\xi_{j}}^{A_{j}}(\theta) \leq h(\theta)$ for every $\theta \in A_{j}$. Hence $h$ and $f_{\xi_{j}}^{A_{j}}$ agree on $A_{j}-d_{j}$. If $d_{j}=\emptyset$, then we set $A_{j+1}=\emptyset$. Clearly $h$ and $f_{\xi_{j}}^{A_{j}}$ agree on $A_{j}-A_{j+1}$. Suppose next $d_{j} \neq \emptyset$. We give $\beta_{j} \in\left[\alpha_{j}, \kappa^{+}\right)$so that for every $\theta \in d_{j}$

$$
f_{\xi_{j}}^{A_{j}}(\theta)<\sup \left(z_{\beta_{j}} \cap \theta\right)<f_{\xi\left(A_{j}, \beta_{j}+1\right)}^{A_{j}}(\theta) .
$$

Recall that $d_{j} \subset A_{j} \subset A_{0}=A\left(x_{\delta}\right) \subset A\left(z_{\delta+1}\right)$ and that $\delta<\alpha_{j}$. Hence $d_{j} \subset A\left(z_{\alpha_{j}}\right)$. Thus by (1) the map $\left\langle\sup \left(z_{\gamma} \cap \theta\right): \gamma \in\left[\alpha_{j}, \kappa^{+}\right)\right\rangle$is increasing and cofinal in $h(\theta)$ for every $\theta \in d_{j}$. Note that $\left|d_{j}\right|<\kappa$ by $d_{j} \subset A\left(x_{\delta}\right)$. Hence by the definition of $d_{j}$ there is $\beta_{j} \in\left[\alpha_{j}, \kappa^{+}\right)$such that the first inequality holds for every $\theta \in d_{j}$. The second inequality holds by (5).

Since $C^{*}$ is club in $\kappa^{+}$, there is $\gamma_{j} \in C^{*}$ such that $\beta_{j}+1<\gamma_{j}$. Define

$$
\begin{aligned}
A_{j+1} & =d\left(f_{\xi\left(A_{j}, \gamma_{j}\right)}^{\left.A_{j}\right)}, f_{\xi\left(A_{j}, \beta_{j}+1\right)}^{A_{j}}\right) \\
& =\left\{\theta \in A_{j}: f_{\xi\left(A_{j}, \gamma_{j}\right)}^{A_{j}}(\theta)<f_{\xi\left(A_{j}, \beta_{j}+1\right)}^{A_{j}}(\theta)\right\} .
\end{aligned}
$$


We claim that $A_{j+1}$ is as required above. By definition $A_{j+1} \subset A_{j}$. Next we show that $f_{\xi_{j}}^{A_{j}}$ and $h$ agree on $A_{j}-A_{j+1}$. Since they agree on $A_{j}-d_{j}$, it suffices to show that $d_{j} \subset A_{j+1}$. We claim that for every $\theta \in d_{j}$

$$
f_{\xi\left(A_{j}, \gamma_{j}\right)}^{A_{j}}(\theta) \leq f_{\xi_{j}}^{A_{j}}(\theta)<f_{\xi\left(A_{j}, \beta_{j}+1\right)}^{A_{j}}(\theta) .
$$

The first inequality follows from $\gamma_{j} \in C^{*}$ and $f_{\xi_{j}}^{A_{j}}(\theta)=\sup \left\{f_{\xi\left(A_{j}, \gamma\right)}^{A_{j}}(\theta): \gamma \in C^{*}\right\}$. The second inequality holds by the choice of $\beta_{j}$. Since $\emptyset \neq d_{j} \subset A_{j+1}$, it remains to give $\gamma<\kappa^{+}$such that

$$
\left\langle A_{i}: i \leq j+1\right\rangle \in T_{\gamma}^{\delta} .
$$

Recall that $\left\langle A_{i}: i \leq j\right\rangle \in T_{\alpha_{j}}^{\delta}$ and that $\alpha_{j}<\beta_{j}+1<\gamma_{j}$. Hence $\left\langle A_{i}: i \leq j\right\rangle \in T_{\gamma_{j}}^{\delta}$. Thus we have $\left\langle A_{i}: i \leq j+1\right\rangle=\left\langle A_{i}: i \leq j\right\rangle *\left\langle d\left(f_{\xi\left(A_{j}, \gamma_{j}\right)}^{A_{j}}, f_{\xi\left(A_{j}, \beta_{j}+1\right)}^{A_{j}}\right)\right\rangle \in T_{\gamma_{j}}^{\delta}$, as desired. This completes the description of the recursion.

It remains to prove that $A_{l+1}=\emptyset$ for some $l<\omega$. Assume to the contrary that $A_{j} \neq \emptyset$ for every $j<\omega$. Note that $\xi\left(A_{j}, \beta_{j}+1\right)<\xi\left(A_{j}, \gamma_{j}\right)$ by $\beta_{j}+1<\gamma_{j}$. Since $f^{A_{j}}$ is increasing in $\prod A_{j}, d\left(f_{\xi\left(A_{j}, \beta_{j}+1\right)}^{A_{j}}, f_{\xi\left(A_{j}, \gamma_{j}\right)}^{A_{j}}\right)$ is in the dual filter of $J^{A_{j}}$. Hence $A_{j+1}=d\left(f_{\xi\left(A_{j}, \gamma_{j}\right)}^{A_{j}}, f_{\xi\left(A_{j}, \beta_{j}+1\right)}^{A_{j}}\right) \in J^{A_{j}}$, i.e. $\max \operatorname{pcf} A_{j+1}<\operatorname{maxpcf} A_{j}$. This is the desired contradiction. $\square$ (Claim 2)

By Claims 1 and 2 we have $z^{*}=w \in S$, as desired. $\mathbf{\text { (Main Claim) }}$

This completes the proof.

\section{REFERENCES}

[1] U. Abraham and M. Magidor, Cardinal arithmetic, Handbook of Set Theory, Springer, Berlin, to appear.

[2] J. Baumgartner And A. Taylor, Saturation properties of ideals in generic extensions. I, Trans. Amer. Math. Soc., 270 (1982), pp. 557-574.

[3] A. Kanamori, The Higher Infinite, Springer Monogr. in Math., Springer, Berlin, 2003.

[4] S. Shelah, Proper Forcing, Lect. Notes in Math., 940, Springer, Berlin, 1982.

[5] - Advances in cardinal arithmetic, Finite and Infinite Combinatorics in Sets and Logic, NATO Adv. Sci. Inst. Ser. C Math. Phys. Sci., 411, pp. 355-383, Kluwer Acad. Publ., Dordrecht, 1993.

[6] _ Cardinal Arithmetic, Oxford Logic Guides, 29, Oxford Univ. Press, New York, 1994.

[7] Strong covering without squares, Fund. Math., 166 (2000), pp. 87-107.

[8] M. SHIOYA, Splitting $\mathcal{P}_{\kappa} \lambda$ into maximally many stationary sets, Israel J. Math., 114 (1999), pp. 347-357.

[9] R. SolOvaY, Strongly compact cardinals and the $G C H$, Proceedings of the Tarski Symposium, Proc. Sympos. Pure Math., XXV, pp. 365-372, Amer. Math. Soc., Providence, 1974. 
M. SHIOYA 